\title{
Association of killer cell immunoglobulin-like receptors with pulmonary tuberculosis in Chinese Han
}

\author{
C. Lu' ${ }^{1}$, Y.-J. Shen ${ }^{1}$, Y.-F. Deng ${ }^{2}$, C.-Y. Wang', G. Fan ${ }^{1}$, Y.-Q. Liu ${ }^{1}$, \\ S.-M. Zhao ${ }^{1}$, B.-C. Zhang ${ }^{1}$, Y.-R. Zhao ${ }^{3}$, Z.-E. Wang ${ }^{1}$, C.-Z. Zhang ${ }^{1}$ \\ and Z.-M. $\mathrm{Lu}^{1}$ \\ ${ }^{1}$ Department of Laboratory Medicine, \\ Shandong Provincial Hospital Affiliated to Shandong University, \\ Shandong University, Jinan, China \\ ${ }^{2}$ Department of Infection Control, Shandong Provincial Chest Hospital, \\ Jinan, China \\ ${ }^{3}$ Department of Center Laboratory, \\ Shandong Provincial Hospital Affiliated to Shandong University, \\ Shandong University, Jinan, China \\ Corresponding author: Z.-M. Lu \\ E-mail: luzhiming@sdu.edu.cn
}

Genet. Mol. Res. 11 (2) 1370-1378 (2012)

Received September 13, 2011

Accepted February 9, 2012

Published May 15, 2012

DOI http://dx.doi.org/10.4238/2012.May.15.7

\begin{abstract}
Killer cell immunoglobulin-like receptors (KIRs) are involved in the pathogenesis of a variety of diseases. However, whether KIR polymorphism is associated with susceptibility to pulmonary tuberculosis was unknown. We examined a possible association of KIR polymorphism with susceptibility to pulmonary tuberculosis in Chinese Han. We analyzed 15 KIR genes in 109 pulmonary tuberculosis patients and 110 healthy controls using sequence-specific primer PCR analysis of genomic DNA. We found that the frequencies of KIR2DS1, 2DS3 and 3DS1 were significantly higher in patients than in the control group. In addition, the number of subjects carrying more than two activating KIR genes in the patient group was significantly higher than in the control group. The gene cluster containing KIR3DS1-
\end{abstract}


2DL5-2DS1-2DS5 was also significantly more frequent in the patient group. In conclusion, KIR genes 2DS1, 2DS3 and 3DS1 appear to be associated with resistance to pulmonary tuberculosis in the Chinese Han population. KIR genes apparently have a role in resistance to pulmonary tuberculosis.

Key words: Pulmonary tuberculosis; Polymorphism; Susceptibility; Killer cell immunoglobulin-like receptor

\section{INTRODUCTION}

Tuberculosis (TB) remains a worldwide health threat. One-third of the world's population is currently infected with Mycobacterium tuberculosis that results in active or latent infection. TB causes nearly 1.8 million deaths annually (Doherty et al., 2009). Because of the increasing mobility of the population, the changing environment, and the biology of bacilli, the prevalence of TB is higher in China. Epidemiological studies indicate that only 5 to $10 \%$ of people infected with M. tuberculosis develop active TB (van Crevel et al., 2002); the immune system can prevent the development of active disease. The genetic factors of TB have been studied using many methods, including selection of candidate genes and genome-wide linkage studies (Casanova and Abel, 2002; Fernando and Britton, 2006; Berrington and Hawn, 2007). Studies focusing on the impact of candidate genes (Zhang et al., 2010) such as human leukocyte antigen (HLA) have provided an understanding of TB infection. Most studies on human genes relevant to pulmonary TB (PTB) have focused on HLA (Sriram et al., 2001). Because KIRs modulate cell function upon recognition of HLA class I molecules, we can infer that they may exert a crucial role in the mechanism of PTB.

KIR belongs to the immunoglobulin superfamily, which is expressed on natural killer (NK) cells and T cells (Parham, 2005). It is named according to the number of extracellular immunoglobulin-like domains (2D or 3D for 2 domains or 3 domains, respectively) and intracytoplasmic tails ( $\mathrm{S}$ or $\mathrm{L}$ for short or long). The family comprises $17 \mathrm{KIR}$ genes, reaching 150 $\mathrm{kb}$ from head to tail on 19q13.4 (Moretta and Moretta, 2004). They are KIR2DL1-5, 2DS1-5, 3DL1-3, 3DS1, 1D, and 2 pseudo genes (3DP1 and 2DP1; Gutiérrez-Rodríguez et al., 2006). Short-tailed receptors confer activating signals with a positively charged amino acid in their transmembrane region. Long-tailed receptors carry immunoreceptor tyrosine-based inhibitory motifs and confer inhibitory signals. Specifically, KIR genes recognize major histocompatibility complex molecules and transmit signals to KIR-bearing cells. A reasonable hypothesis is that KIR polymorphic loci regulate host immunity against infectious pathogens (Yen et al., 2001). Moreover, accumulating evidence suggests that KIR-specific genes contribute to the pathogenesis of other diseases (Cooley et al., 2009; Vidal-Castineira et al., 2010; Seich Al Basatena et al., 2011), such as psoriasis, rheumatoid arthritis, hepatitis C. However, the functions and effects of KIR genes during the development of TB remain unclear in the Chinese population.

In the present study, we aimed to investigate the KIR genes in PTB patients and controls using sequence-specific primer polymerase chain reaction (PCR). The innovative point of our study was the subdivision of the patient group according to sputum smear test (positive and negative). Our findings provide a better understanding of the genetic diversity of KIR genes across PTB patients in China. 


\section{MATERIAL AND METHODS}

\section{Subjects}

A total of 109 patients (60 men and 49 women; mean age: 41.7 years; range: 19-69 years) with PTB and 110 unrelated healthy controls (53 men and 57 women; mean age: 38.3 years; range: 21-67 years) were recruited from Shandong Chest Hospital, Jinan, between May 2010 and May 2011. The patients were treated at the hospital. According to American Tuberculosis Society criteria, patients were diagnosed on the basis of history, presence of recent clinical symptoms, clinical signs, chest radiography, tuberculin skin test, and sputum smear test. No extra-pulmonary involvement was detected in the patients. The patient group was subdivided into two groups according to the results of a sputum smear test (positive or negative). The average age of the subgroups was the same. Blood was collected before the initiation of anti-tuberculosis therapy. A total of 110 unrelated healthy subjects with a history of positive (indurate area $\geq 10 \mathrm{~mm}$ with vaccination scars) tuberculin skin test $(0.1 \mathrm{~mL}$ purified protein derivative in a concentration of $5 \mathrm{U}$ ) were recruited as controls. Radiographic scans of controls revealed no PTB, and all subjects had been inoculated with Bacillus Calmette-Guerin vaccine.

Patients and controls were matched for age, gender, ethnicity, and socioeconomic status. Patients and controls with diabetes, chronic renal failure, malignant disease, and immunological or autoimmune disease as well as those who were human immunodeficiency virus positive or had other concurrent diseases that could affect the immune system were excluded. Informed consent was obtained from each individual. The Hospital Ethics Committee approved the research.

\section{Genomic DNA isolation}

Genomic DNA was extracted from $5 \mathrm{~mL}$ ethylenediaminetetraacetic acid anticoagulated peripheral blood using a TIANamp Blood DNA Kit (Tiangen Biotech, Beijing, China) according to the manufacturer's instructions, and the extracted DNA was stored at $-20^{\circ} \mathrm{C}$. The integrity and quantity of DNA samples were determined using a UV spectrophotometer, and the DNA concentration was adjusted to $50 \mathrm{ng} / \mu \mathrm{L}$.

\section{Sequence-specific primer PCR}

All recruited subjects underwent KIR genotyping. Our previous research demonstrated that the framework genes KIR2DL4, 3DL2, 3DL3, and 3DP1 are present in all individuals (Zhi-Ming et al., 2007). Therefore, KIR locus typing was performed to detect the presence or absence of 11 known KIR genes, including 2DL1-3, 2DL5, 2DS1-5, 3DL1, and 3DS1. The primers used in this study were designed based on primer sites described elsewhere (Martin et al., 2002b). All primers (Bo Ya Biotechnology Co. Ltd., Shanghai, China) were validated, and their gene specificity was confirmed. Two sets of primers were designed for each KIR gene (except for 2DS5; Table 1). PCR was preformed within a $20-\mu \mathrm{L}$ system containing $6.6 \mu \mathrm{L}$ PCR loading dye mix (Takara, Kyoto, Japan) including Taq DNA polymerase, $6 \mu \mathrm{L}$ forward and reverse primers $(1.2 \mu \mathrm{M}), 6.9 \mu \mathrm{L}$ RNase Free (Takara), and $0.5 \mu \mathrm{L}$ genomic DNA (50 ng/ $\mu \mathrm{L}$ ). Briefly, after a denaturing step at $94^{\circ} \mathrm{C}$ for $1 \mathrm{~min}, \mathrm{PCR}$ amplification was carried out with 10 
cycles at a melting temperature of $94^{\circ} \mathrm{C}$ for $30 \mathrm{~s}$, and an annealing temperature of $65^{\circ} \mathrm{C}$ for 30 s. Subsequently, 20 cycles were performed at a melting temperature of $94^{\circ} \mathrm{C}$ for $30 \mathrm{~s}$, an annealing temperature of $62^{\circ} \mathrm{C}$ for $30 \mathrm{~s}$, and an extension temperature of $72^{\circ} \mathrm{C}$ for $40 \mathrm{~s}$. Finally, an extra extension step at $72^{\circ} \mathrm{C}$ was preformed. The amplicons were analyzed on ethidium bromide-stained agarose gels (1.5\%) using a 1-kb DNA ladder as a molecular weight marker. After electrophoresis, the agarose gel was scanned and imaged with an Alphaimager TM 2200 instrument (Alpha Innotech Corporation, San Leandro, CA, USA). Products of predicted size were visualized under ultraviolet light, and each sample was genotyped.

\begin{tabular}{llll}
\multicolumn{2}{l}{ Table 1. SSP-PCR primers of KIR genes. } & & \\
\hline & & & Bp \\
\hline KIR & Forward (5'-3') & Reverse (5'-3') & 197 \\
\hline 3DL1-1 & CGCTGTGGTGCCTCGA & GGTGTGAACCCCGACATG & 181 \\
3DL1-2 & CCCTGGTGAAATCAGGAGAGAG & TGTAGGTCCCTGCAAGGGCAA & 300 \\
3DS1-1 & AGCCTGCAGGGAACAGAAG & GCCTGACTGTGGTGCTCG & 177 \\
3DS1-2 & CCTGGTGAAATCAGGAGAGAG & GTCCCTGCAAGGGCAC & 214 \\
2DL5-1 & GCGCTGTGGTGCCTCG & GACCACTCAATGGGGGAGC & 194 \\
2DL5-2 & TGCAGCTCCAGGAGCTCA & GGGTCTGACCACTCATAGGGT & 127 \\
2DL1-1 & GTTGGTCAGATGTCATGTTTGAA & GGTCCCTGCCAGGTCTTGCG & 330 \\
2DL1-2 & TGGACCAAGAGTCTGCAGGA & TGTTGTCTCCCTAGAAGACG & 173 \\
2DL2-1 & CTGGCCCACCCAGGTCG & GGACCGATGGAGAAGTTGGCT & 150 \\
2DL2-2 & GAGGGGGAGGCCCATGAAT & TCGAGTTTGACCACTCGTAT & 550 \\
2DL3-1 & CTTCATCGCTGGTGCTG & AGGCTCTTGGTCCATTACAA & 800 \\
2DL3-2 & TCCTTCATCGCTGGTGCTG & GGCAGGAGACAACTTTGGATCA & 102 \\
2DS2-1 & TTCTGCACAGAGAGGGGAAGTA & CTTCTCCATCAGTCGCATGAG & 240 \\
2DS2-2 & CGGGCCCCACGGTTT & GGTCACTCGAGTTTGACCACTCA & 242 \\
2DS3-1 & TGGCCCACCCAGGTCG & TGAAAACTGATAGGGGGAGTGAGG & 190 \\
2DS3-2 & CTATGACATGTACCATCTATCCAC & AAGCAGTGGGTCACTTGAC & 204 \\
2DS4-1 & CTGGCCCTCCCAGGTCA & TCTGTAGGTTCCTGCAAGGACAG & 2000 \\
2DS4-2 & CTGGCCCTCCCAGGTCA & GGAATGTTCCGTTGATGC & 125 \\
2DS5 & TGATGGGGTCTCCAAGGG & TCCAGAGGGTCACTGGGC & 102 \\
2DS1-1 & CTTCTCCATCAGTCGCATGAA & CTTCTCCATCAGTCGCATGAG & 102 \\
2DS1-2 & CTTCTCCATCAGTCGCATGAA & AGAGGGTCACTGGGAGCTGAC & \\
\hline & & & \\
\end{tabular}

\section{Statistical analysis}

Briefly, observed phenotype frequencies ( $p f)$ of KIR genes were determined using the ratio of gene presence within the population over the total population. Genotype frequency $(g f)$ of each locus was calculated using following formula: genotype frequency $=1-\sqrt{(1-p f)}$. Framework genes were included in the analysis of KIR gene numbers and their ratios. Frequency differences of KIR loci between patients and controls were analyzed using the chi-square test. The $95 \%(95 \% \mathrm{CI})$ confidence interval of the calculated odd ratio (OR) was estimated. P value was multiplied by the number of comparisons, and a value of $\mathrm{P}<0.05$ was considered statistically significant. Analyses were performed using Statistical Package for Social Sciences Version 16.0 (SPSS, Chicago, IL, USA).

\section{RESULTS}

Statistical analysis indicated no significant differences between groups with respect to age and gender $(\mathrm{P}>0.05)$. All KIR genes were tested both in the patient groups (smear negative and positive) and in the control group at different frequencies (Table 2). Figure 1 shows the amplification of KIR genes from one patient. 


\begin{tabular}{|c|c|c|c|c|c|c|c|c|}
\hline \multirow[t]{2}{*}{ KIR } & \multicolumn{3}{|c|}{ Control $(\mathrm{N}=110)$} & \multicolumn{3}{|c|}{ PTB $(\mathrm{N}=109)$} & \multirow[t]{2}{*}{$\mathrm{OR}(\mathrm{CI})$} & \multirow[t]{2}{*}{$P$} \\
\hline & $\mathrm{n}$ & $p f(\%)$ & $g f(\%)$ & $\mathrm{n}$ & $p f(\%)$ & $g f(\%)$ & & \\
\hline \multicolumn{9}{|c|}{ Inhibitory } \\
\hline 2DL1 & 107 & 97.3 & 83.6 & 108 & 99.1 & 90.5 & $3.028(0.310 \sim 29.572)$ & 0.317 \\
\hline 2DL2 & 73 & 66.4 & 42.0 & 83 & 76.1 & 51.1 & $1.618(0.895 \sim 2.925)$ & 0.110 \\
\hline 2DL3 & 105 & 95.5 & 78.8 & 108 & 99.1 & 90.5 & $5.143(0.591 \sim 44.763)$ & 0.100 \\
\hline 2DL5 & 77 & 70.0 & 45.2 & 78 & 71.6 & 46.7 & $1.078(0.602 \sim 1.931)$ & 0.800 \\
\hline 3DL1 & 108 & 98.2 & 86.6 & 108 & 99.1 & 90.5 & $2.000(0.179 \sim 22.385)$ & 0.566 \\
\hline \multicolumn{9}{|c|}{ Activating } \\
\hline $2 \mathrm{DS} 1$ & 38 & 34.5 & 19.1 & 60 & 55.0 & 32.9 & $2.320(1.345 \sim 4.000)$ & $0.002 *$ \\
\hline 2DS2 & 37 & 33.6 & 18.5 & 40 & 36.7 & 20.4 & $1.144(0.657 \sim 1.993)$ & 0.635 \\
\hline $2 \mathrm{DS} 3$ & 21 & 19.1 & 10.1 & 50 & 45.9 & 26.4 & $3.052(1.958 \sim 6.588)$ & $<0.001^{*}$ \\
\hline 2DS4 & 101 & 91.8 & 71.4 & 105 & 96.3 & 80.8 & $2.339(0.698 \sim 7.837$ & 0.158 \\
\hline 2DS5 & 39 & 35.5 & 19.7 & 36 & 33.0 & 18.1 & $0.898(0.514 \sim 1.569)$ & 0.705 \\
\hline $3 \mathrm{DS} 1$ & 56 & 50.9 & 30.0 & 74 & 67.9 & 43.3 & $2.039(1.177 \sim 3.530)$ & $0.011 *$ \\
\hline
\end{tabular}

$\mathrm{KIR}=$ Killer cell immunoglobulin-like receptor; $\mathrm{PTB}=$ pulmonary tuberculosis. $\mathrm{P}$ value $=$ comparison between healthy control group and PTB group. P values were determined using chi-square test. $* \mathrm{P}<0.05$.

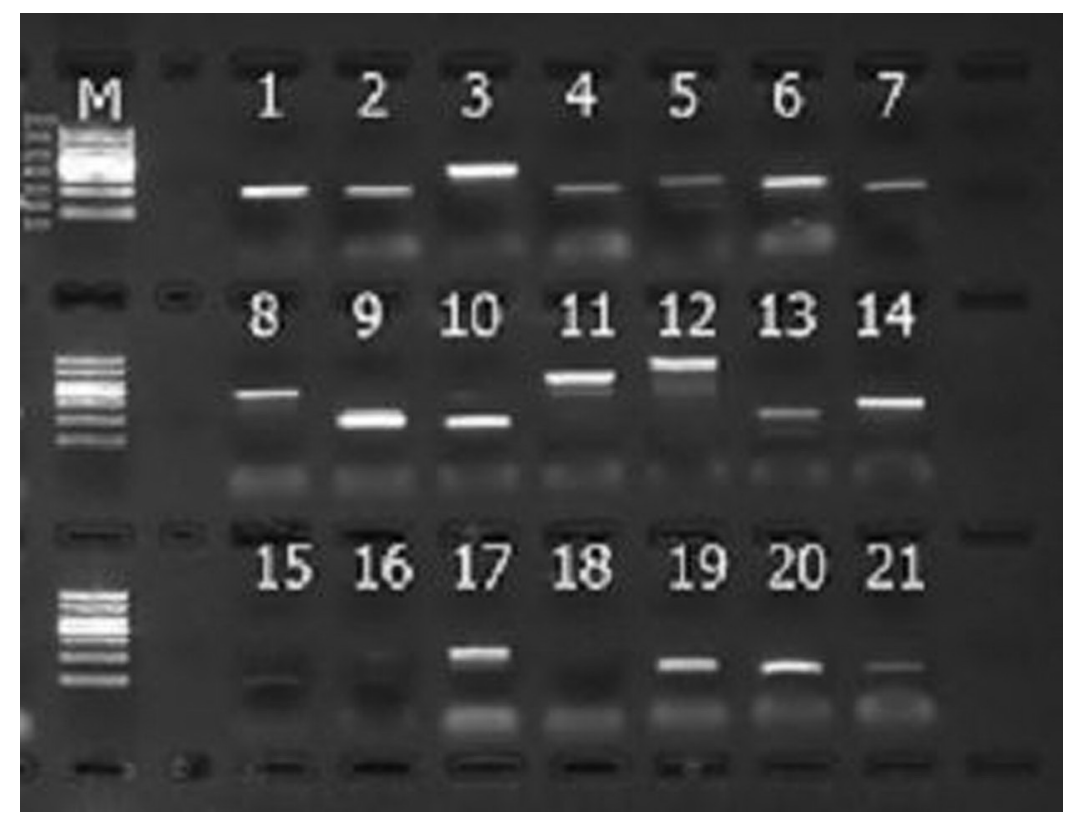

Figure 1. The patient was positive for all KIR genes except for KIR2DS3 $(15,16)$. The corresponding digits represent: 1,2 - KIR3DL1; 3,4 - KIR2DL5; 5,6 - KIR3DS1; 7,8 - KIR2DL1; 9,10 - KIR2DL2; 11,12 - KIR2DL3; 13,14 - KIR2DS2; 15,16 - KIR2DS3; 17,18 - KIR2DS4; 19 - KIR2DS5; 20,21 - KIR2DS1; M-DNA marker.

\section{Phenotype and genotype frequencies of KIRs in patients and controls}

Our data showed that KIR3DL1, 2DL1, 2DL3, and 2DS4 were the most frequently found genes in both patients and controls. The total carriage frequencies of KIR2DS1, 2DS3, and 3DS1 in the patient groups were higher than those in the control group $(\mathrm{P}=0.002, \mathrm{P}<$ 0.001 , and $\mathrm{P}=0.011$, respectively). Moreover, the frequencies of KIR2DS2 and KIR2DS5 in 
the smear-negative group were higher than those in the smear-positive group $(\mathrm{P}=0.001$ and $\mathrm{P}$ $<0.001$, respectively; Figure 2).

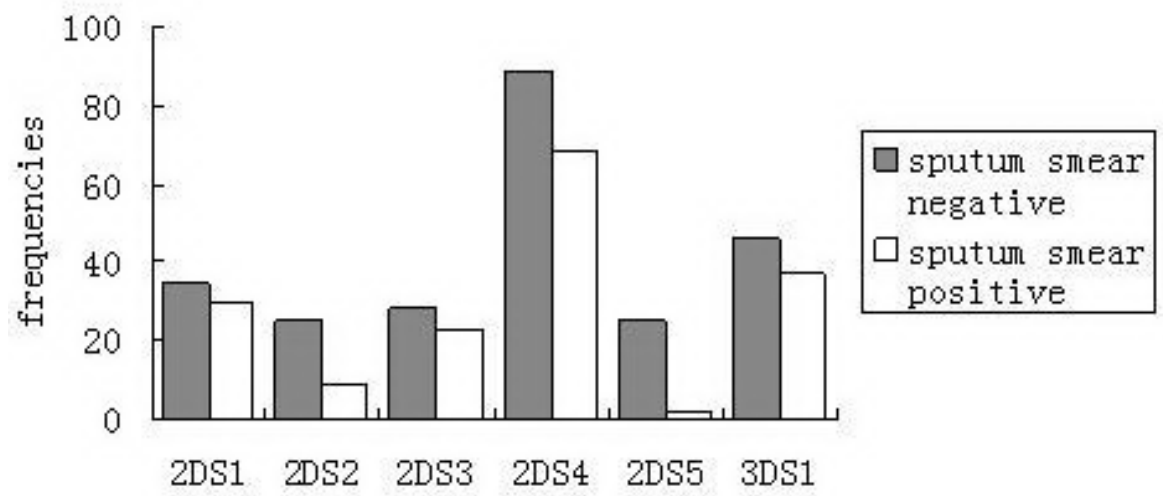

Figure 2. The frequencies of activating KIRs between sputum smear negative group and positive group.

\section{Activating KIR genes in patients and controls}

To analyze our data further, we categorized patients and controls into two groups: Group 1 included subjects carrying less than two activating KIR genes. Group 2 included subjects carrying two or more activating KIR genes (Table 3). Of 109 patients, 20.2 and 79.8\% were categorized into group 1 and group 2, respectively. On the contrary, 32.7 and $67.3 \%$ of controls were categorized into group 1 and group 2, respectively. Moreover, we found that genotypes containing six activating KIR genes were significantly more frequent in the patient group than in the control group (11.00 vs $1.80 \%, \mathrm{P}=0.005)$.

Table 3. The differences between the two groups in activating gene numbers.

\begin{tabular}{|c|c|c|c|c|c|c|}
\hline \multirow{2}{*}{$\begin{array}{l}\text { Activating } \\
\text { KIRs }\end{array}$} & \multicolumn{2}{|c|}{ Control $(\mathrm{N}=110)$} & \multicolumn{2}{|c|}{ PTB $(\mathrm{N}=109)$} & \multirow[t]{2}{*}{$\mathrm{P}$} & \multirow[t]{2}{*}{$\mathrm{OR}(\mathrm{CI})$} \\
\hline & + & $\mathrm{f}(\%)$ & + & $\mathrm{f}(\%)$ & & \\
\hline Less than two & 36 & 32.7 & 22 & 20.2 & $0.035^{*}$ & $0.520(0.281 \sim 0.961)$ \\
\hline Two or more than two & 74 & 67.3 & 87 & 79.8 & $0.035^{*}$ & $0.520(0.281 \sim 0.961)$ \\
\hline
\end{tabular}

+ - positive case numbers. $\mathrm{P}$ value was determined using chi-square test. ${ }^{*} \mathrm{P}<0.05$.

\section{Increased frequency of one gene cluster in the patient group}

Based on the linkage disequilibrium between certain alleles of various KIR loci, the frequency of one gene cluster containing KIR3DS1-2DL5-2DS1-2DS5 was significantly increased in patients compared with controls $(30.3 v s 15.5 \%, \mathrm{P}=0.009)$.

\section{DISCUSSION}

Genetic diversity is known to play an important role in PTB. Recent evidence has suggested that $\mathrm{T}$ cells expressing KIR2DS2 can mediate vascular damage in patients with 
rheumatoid arthritis, implicating a role of activating KIR genes in rheumatoid arthritis and other autoimmune diseases (Williams et al., 2005). Previous studies have indicated that HLA confers susceptibility to TB (Pospelov et al., 2007). In addition, some KIR genes such as 3DS1 can bind to HLA-B27 and recruit positive signals, resulting in NK cell activation (Lanier, 1998). This recognition can affect immunomodulatory functions of NK cells (Campbell and Purdy, 2011). Martin et al. (2002a) have reported that the activating KIR3DS1 in combination with HLA-B alleles is associated with delayed progression to acquired immunodeficiency syndrome in individuals infected with human immunodeficiency virus type 1.

In our present study, the diversity of 15 KIR genes in 109 PTB patients and 110 controls was studied to assess the effect of KIR polymorphisms on the susceptibility to PTB. The imbalance between activating and inhibitory KIRs may affect the activation of immune cells, contributing to the pathogenesis of diseases. KIR2DS1, 2DS3, and 3DS1 may serve as PTB susceptibility genes. Our results were inconsistent with data described by Mendez et al. (2006) showing that KIR2DL3 is significant in patients compared with controls, however. The discrepancy can be explained by the fact that they investigated a different population-each population has a specific KIR distribution. According to data from Jiang et al. (2005), the gene frequency of KIR2DL3 is more than $90 \%$ in the Chinese Han population; therefore, KIR2DL3 was not significant between groups in our present study. The sample size, diversity of technology used, linkage disequilibrium, and other limitations in our study design might also explain the discrepancy between our data and those of Mendez et al. (2006).

Smear-positive patients are the main source of infection in a community. Only $10 \%$ of individuals develop clinical disease. The immune response in the remaining individuals only succeeds in containing the infection, as some bacilli escape killing by the mechanisms of immune activity and remain in latent or dormant states. Clinically, the frequencies of KIR2DS2 and KIR2DS5 in the smear-positive group were lower than those in the smear-negative group. These results suggest that activating KIRs may be involved in PTB susceptibility and the regulation of clinical evolution during disease development. Therefore, the genetic imbalance between activating and inhibitory KIR genes is necessary for the development of PTB. Mycobacterium tuberculosis is an intracellular pathogen that can persist within the host. Infection and antibody production can lead to chronic or fatal disease. KIRs can recognize pathogenassociated molecules and contribute to immune responses. NK cells play a crucial role in mediating innate immunity (Biron et al., 2002), and T cells primarily mediate the acquired immunity to pathogens, such as protecting against M. tuberculosis infection (Brunstein et al., 2009). The important point for the development of immunity against TB involves the engagement of $\mathrm{CD}^{+}$and $\mathrm{CD} 8^{+}$lymphocyte subpopulations. $\mathrm{CD} 4^{+} \mathrm{T}$ cells participate in the amplification and regulation of immunity by producing cytokines. $\mathrm{CD} 8^{+} \mathrm{T}$ cells act as cytotoxic effectors that can break the infected target cells (Rodrigues et al., 2002). Mounting evidence suggests that KIR gene diversity determines susceptibility to infectious diseases by sending inhibitory or activating signals. In our study, the increased presence of activating KIRs in patients with PTB may affect immune response. More KIR genes send activating signals to NK cells and T cells in response to M. tuberculosis.

Taken together, our results suggest that susceptibility to PTB is mediated by complex interactions between factors: (1) Each population has a specific KIR distribution. The polymorphism between ethnicity and geographical location has been described for various ethnic diversities (Norman et al., 2001; Gutiérrez-Rodríguez et al., 2006). (2) KIR genes are orga- 
nized into a highly polymorphic, multi-gene family with considerable allelic polymorphism. The interaction between KIR genes and other multiple genes may confer susceptibility to PTB. (3) The frequency of one gene cluster containing KIR3DS1-2DL5-2DS1-2DS5 was significantly increased in the patient group. Therefore, linkage disequilibrium between different KIR loci may play an important role in disease development. (4) Differences in environmental and cultural factors along with gene-gene interactions and even variations in M. tuberculosis strains complicate the understanding of the observed differences. Our findings expand the horizon of elucidating KIR gene diversity and add another level to the complex mechanisms of NK cell/T cell-mediated response to PTB infection. Moreover, this study provides additional understanding of KIR genes on which to base future investigations of PTB. Further functional study of KIR genes will be helpful for clarifying the mechanism of PTB susceptibility and providing new approaches for the rational design of vaccines and immune therapies.

\section{ACKNOWLEDGMENTS}

Research supported by the Shandong Provincial Scientific and Technological Development Projects Foundation (2009GG10002014) and Chinese National Natural Science Foundation (grant 30371304). We thank Yu-Lian Jiao, Xue-Jin Gao, and others working in the Department of Center Laboratory of Shandong Provincial Hospital for their skillful technical help.

\section{REFERENCES}

Berrington WR and Hawn TR (2007). Mycobacterium tuberculosis, macrophages, and the innate immune response: does common variation matter? Immunol. Rev. 219: 167-186.

Biron CA, Nguyen KB and Pien GC (2002). Innate immune responses to LCMV infections: natural killer cells and cytokines. Curr. Top. Microbiol. Immunol. 263: 7-27.

Brunstein CG, Wagner JE, Weisdorf DJ, Cooley S, et al. (2009). Negative effect of KIR alloreactivity in recipients of umbilical cord blood transplant depends on transplantation conditioning intensity. Blood 113: 5628-5634.

Campbell KS and Purdy AK (2011). Structure/function of human killer cell immunoglobulin-like receptors: lessons from polymorphisms, evolution, crystal structures and mutations. Immunology 132: 315-325.

Casanova JL and Abel L (2002). Genetic dissection of immunity to mycobacteria: the human model. Annu. Rev. Immunol. 20: 581-620.

Cooley S, Trachtenberg E, Bergemann TL, Saeteurn K, et al. (2009). Donors with group B KIR haplotypes improve relapse-free survival after unrelated hematopoietic cell transplantation for acute myelogenous leukemia. Blood 113: 726-732.

Doherty M, Wallis RS and Zumla A (2009). Biomarkers for tuberculosis disease status and diagnosis. Curr. Opin. Pulm. Med. 15: 181-187.

Fernando SL and Britton WJ (2006). Genetic susceptibility to mycobacterial disease in humans. Immunol. Cell Biol. 84: 125-137.

Gutiérrez-Rodríguez ME, Sandoval-Ramirez L, Diaz-Flores M, Marsh SG, et al. (2006). KIR gene in ethnic and Mestizo populations from Mexico. Hum. Immunol. 67: 85-93.

Jiang K, Zhu FM, Lv QF and Yan LX (2005). Distribution of killer cell immunoglobulin-like receptor genes in the Chinese Han population. Tissue Antigens 65: 556-563.

Lanier LL (1998). NK cell receptors. Аnпи. Rev. Immunol. 16: 359-393.

Martin MP, Gao X, Lee JH, Nelson GW, et al. (2002a). Epistatic interaction between KIR3DS1 and HLA-B delays the progression to AIDS. Nat. Genet. 31: 429-434.

Martin MP, Nelson G, Lee JH, Pellett F, et al. (2002b). Cutting edge: susceptibility to psoriatic arthritis: influence of activating killer Ig-like receptor genes in the absence of specific HLA-C alleles. J. Immunol. 169: 2818-2822.

Mendez A, Granda H, Meenagh A, Contreras S, et al. (2006). Study of KIR genes in tuberculosis patients. Tissue Antigens 68: 386-389. 
Moretta L and Moretta A (2004). Killer immunoglobulin-like receptors. Curr. Opin. Immunol. 16: 626-633.

Norman PJ, Stephens HA, Verity DH, Chandanayingyong D, et al. (2001). Distribution of natural killer cell immunoglobulinlike receptor sequences in three ethnic groups. Immunogenetics 52: 195-205.

Parham P (2005). MHC class I molecules and KIRs in human history, health and survival. Nat. Rev. Immunol. 5: 201-214.

Pospelov LE, Matrakshin AG, Malenko AF, Udina IG, et al. (2007). Genetic HLA markers associated with pulmonary tuberculosis in the Barum-Khemchiksky District, Republic of Tyva. Probl. Tuberk. Bolezn. Legk. 62-64.

Rodrigues DS, Medeiros EA, Weckx LY, Bonnez W, et al. (2002). Immunophenotypic characterization of peripheral T lymphocytes in Mycobacterium tuberculosis infection and disease. Clin. Exp. Immunol. 128: 149-154.

Seich Al Basatena NK, Macnamara A, Vine AM, Thio CL, et al. (2011). KIR2DL2 enhances protective and detrimental HLA class I-mediated immunity in chronic viral infection. PLoS Pathog. 7: e1002270.

Sriram U, Selvaraj P, Kurian SM, Reetha AM, et al. (2001). HLA-DR2 subtypes \& immune responses in pulmonary tuberculosis. Indian J. Med. Res. 113: 117-124.

van Crevel R, Ottenhoff TH and van der Meer JW (2002). Innate immunity to Mycobacterium tuberculosis. Clin. Microbiol. Rev. 15: 294-309.

Vidal-Castineira JR, Lopez-Vazquez A, Diaz-Pena R, Alonso-Arias R, et al. (2010). Effect of killer immunoglobulin-like receptors in the response to combined treatment in patients with chronic hepatitis $\mathrm{C}$ virus infection. J. Virol. 84: 475-481.

Williams F, Meenagh A, Sleator C, Cook D, et al. (2005). Activating killer cell immunoglobulin-like receptor gene KIR2DS1 is associated with psoriatic arthritis. Hum. Immunol. 66: 836-841.

Yen JH, Moore BE, Nakajima T, Scholl D, et al. (2001). Major histocompatibility complex class I-recognizing receptors are disease risk genes in rheumatoid arthritis. J. Exp. Med. 193: 1159-1167.

Zhang YX, Xue Y, Liu JY and Zhao MY (2010). Association of TIRAP (MAL) gene polymorphisms with susceptibility to tuberculosis in a Chinese population. Genet. Mol. Res. 10: 7-15.

Zhi-ming L, Yu-lian J, Zhao-lei F, Chun-xiao W, et al. (2007). Polymorphisms of killer cell immunoglobulin-like receptor gene: possible association with susceptibility to or clearance of hepatitis B virus infection in Chinese Han population. Croat. Med. J. 48: 800-806. 